В.Анпилогов, К.т.Н., заместитель генерального директора АО "ВИСАТ-ТЕЛ" / avr@visat.tel, Нгуен Дык Ань, аспирант МФТИ / anhforce@gmail.com

УДК 004.735, DOI: 10.22184/2070-8963.2020.91.6.44.53

Представлены технические параметры наиболее известных технологий LPWAN, большая часть которых относится к проприетарным. Oсобое место занимает LoRa - фактически единственная открытая технология. Как показал анализ возможности адаптации технологий LPWAN для использования в спутниковых сетях ІоТ, ориентированных на прямой доступ к оконечным устройствам, наиболее перспективная технология - LoRa. B статье приводятся графические данные взаимосвязи отношения сигнал/ шум и вероятности ошибки на бит, которые обеспечивают объективную оценку бюджета абонентских радиолиний спутниковых каналов, реализованных на основе технологии LoRa.

История Интернета вещей началась много десятилетий назад. Еще в 1970-х годах была сформулирована задача автоматизации производственных и технологических процессов с использованием систем передачи данных, которые назвали автоматическими системами управления технологическими процессами (АСУ ТП). С появлением интернета практическая ценность таких систем принципиально повысилась. Примерно с начала 2010-х годов в обиход вошло словосочетание "Интернет вещей".

Специфика спутниковых систем управления и контроля обусловлена их глобальностью. В начале 2000-х годов в качестве составной части АСУ ТП предлагались спутниковые решения SCADA (Sup ervisory Control And Data Acquisition - диспетчерское управление и сбор данных) на основе технологии VSAT с узкополосными каналами. Но еще раньше, в конце 1980-х годов, появилось понятие межмашинного взаимодействия М2M с использованием спутниковых систем. Первой такой системой, по-видимому, следует считать OmniTRACS, созданную в 1988 году. Через несколько лет, в 1991-м, компания Eutelsat реализовала аналогичный проект Euteltracs. Эти системы были ориентированы на отслеживание и диспетчеризацию грузовых перевозок.
С появлением в начале 2000-х годов спутниковых систем подвижной спутниковой службы (ПСС) Iridium, Globalstar и др. услуги М2М стали одной из их дополнительных функций. Однако ни по ценовым, ни по эксплуатационным параметрам они не могут конкурировать с аналогичными услугами в наземных сетях [1-3], даже после глубокой модернизации систем ПСС.

Поскольку бесперспективность систем ПСС очевидна, формируется новый сегмент спутниковой связи, названный спутниковым Интернетом вещей (спутниковым IоT). Этот сегмент охватывает все функции и услуги, которые ранее обозначались как ACУ TП, SCADA и M2M, а также задачи из области промышленного IoT (IIoT) и даже Интернета всего (IoE). Общее количество подключаемых устройств, по всем прогнозам, составит десятки миллиардов уже в ближайшие пять лет. Но применительно к спутниковому IоT не стоит скатываться в примитивизм с подключением холодильников, чайников и т.п. Хотя одна из ключевых задач спутникового ІоТ - обеспечение ценовых и эксплуатационных параметров абонентского оборудования и услуг, соизмеримых с аналогами в наземных сетях LPWAN и сотовых сетях. Очевидно, что потребителя не интересует, какие физические каналы используются, 
если достигнуто равенство ценовых показателей и эксплуатационных параметров при решении его задач. В данном случае потенциальный рынок спутникового IоT - это общий рынок Интернета вещей. Однако очевидно, что такого паритета для всех сегментов рынка достичь невозможно, как и, например, создать всеобъемлющие сети 5G, даже в отдаленной перспективе. У каждой технологии свой целевой рынок.

Потребительский рынок услуг спутникового IoT анализируется в ряде отчетов компании NSR [4], но относиться к прогнозным результатам следует критически, поскольку выводы о развитии данного рынка базируются на анализе действующих систем ПСС. Альтернативные исследования [5] показывают, что рынок спутникового ІоТ по количеству прогнозируемых подключений может быть больше примерно в 50 раз, а его потенциальный объем - в четыре раза (примерно 10-12 млрд долл.) по сравнению с данными из прогнозов NSR. Ho эти параметры могут быть достигнуты только при ценовых показателях услуги и абонентских устройств, соизмеримых с показателями, характерными для сетей LPWAN и сотовых сетей, в сегментах, где ликвидация ограничений рабочей зоны и тем более глобальность услуги - важные условия.

Решение этой задачи во многом зависит от технологии каналообразования, применяемой в спутниковой сети IоT. Следует отметить, что в данном случае рассматривается способ организации сети, в которой обеспечивается прямой доступ к оконечным устройствам IоT по аналогии с сетями LPWAN. Поэтому необходим объективный анализ технологий LPWAN для оценки возможности их адаптации к спутниковой сети IоT.

\section{ТЕХНОЛОГИИ LPWAN}

Современные технологии сетей IоT следует разделить на две самостоятельные группы. К первой относятся технологии, которые могут быть реализованы только при использовании полной инфраструктуры сетей сотовой связи (2G/3G/4G/5G). Наибольшую популярность получила технология NB-IoT [6]. Эта группа технологий не подходит для организации спутниковых сетей с прямым доступом к оконечным абонентским устройствам IоT. В таком случае спутниковый канал применяется как обычный backhaul [7]. Очевидно, что достичь паритета по ценовым показателям услуги в такой спутниковой сети IоТ невозможно, поскольку сегмент сотовой сети дополняется дорогостоящим спутниковым каналом.
Во вторую группу входят технологии LPWAN, на основе которых создаются самостоятельные (выделенные) сети с целевой функцией Интернета вещей, обеспечивающие прямой доступ к оконечным устройствам IоT. Адаптация технологии LPWAN применительно к таким системам спутникового IоT представляет наибольший интерес, поскольку не требуется полной инфраструктуры сотовой сети. Однако у каждой технологии LPWAN свои особенности, которые, в конечном итоге, сказываются на возможности и/или эффективности их адаптации для спутниковой системы, ориентированной на обеспечение прямого доступа к оконечным устройствам IоT.

В табл.1 представлен ряд технологий LPWAN, которые предназначены для работы в диапазонах ISM ниже 1 ГГц, и их типовые технические параметры. Необходимо особо отметить, что указанные технические параметры не отражают всего многообразия их вариантов, следующих из официальных описаний технологии. Привести в статье все варианты невозможно, поэтому в табл.1 собраны параметры, соизмеримые между собой по энергетическим показателям и достаточные для количественных оценок эффективности технологий LPWAN. Следует учитывать, что не все технологии LPWAN имеют достаточное для анализа описание в открытых информационных источниках. Иногда указанные в них описания и параметры противоречивы, поэтому возможны разночтения с иными публикуемыми материалами. Некоторые технологии LPWAN упоминаются в массовых изданиях, но не имеют открытых технических описаний (или авторам они неизвестны), поэтому не представлены в табл.1 (например, Ingenu (RPMA), модификация LoRaWAN: Symphony Link и др.).

Среди технологий LPWAN можно выделить две группы. Первая - ультра "узкополосные технологии", вторая группа, которая представлена только технологией LoRaWAN и ее модификацией Symphony Link, - это условно "широкополосная" LPWAN.

Возможна классификация технологий LPWAN и по требованию синхронизации в сети, то есть протоколы асинхронные и синхронные.

У каждой технологии LPWAN свои особенности и приоритетные области использования при формировании сервисов IоT. Но общее для них свойство - это, как правило, возможность обеспечить работоспособность оконечных устройств от обычной бытовой батарейки в течение многих лет при очень низких ценных параметрах оборудования [8]. При этом передавать полезную 
Таблица 1. Технические параметры технологий LPWAN

\begin{tabular}{|c|c|c|c|c|c|c|c|c|c|}
\hline Параметр & LoRa & SigFox & NB-Fi & XNB & OpenUNB & Weightless-P & SNBWAN & DASH7 & $\begin{array}{c}\text { Mioty } \\
\text { (Class A) }\end{array}$ \\
\hline $\begin{array}{l}\text { Полоса частот } \\
\text { канала "вверх", } \\
\text { кГц }\end{array}$ & \multirow[t]{2}{*}{125} & 0,1 & \multirow[t]{2}{*}{0,05} & \multirow[t]{2}{*}{0,1} & \multirow[t]{2}{*}{0,1} & 12,5 & \multirow{2}{*}{\begin{tabular}{|l}
$0,4 / 0,8 /$ \\
$6,4 / 51,2 /$ \\
204,8
\end{tabular}} & \multirow[t]{2}{*}{25} & \multirow[t]{2}{*}{2,38} \\
\hline $\begin{array}{l}\text { Полоса частот } \\
\text { канала "вниз", кГц }\end{array}$ & & 1,5 & & & & 100 & & & \\
\hline $\begin{array}{l}\text { Метод разделения } \\
\text { каналов Tx/Rx }\end{array}$ & TDD & FDD & TDD & FDD & FDD & & TDD & & FDD \\
\hline $\begin{array}{l}\text { Число каналов } \\
\text { "вверх" (максимум) }\end{array}$ & 8 & 1920 & \multirow{2}{*}{1024} & \multirow{2}{*}{5000} & \multirow{2}{*}{ Н.д. } & $\begin{array}{c}8 \\
\text { (по } 12,5 \text { кГц }) \\
\end{array}$ & \multirow{2}{*}{ До 512} & 270 (EU) & \multirow{2}{*}{42} \\
\hline $\begin{array}{l}\text { Число каналов } \\
\text { "вниз" (максимум) }\end{array}$ & 1 & 128 & & & & 1 & & & \\
\hline $\begin{array}{l}\text { Модуляция на } \\
\text { линии "вверх" }\end{array}$ & $\begin{array}{c}\text { ЛЧМ, } \\
\mathrm{SF}=6 \ldots 12 \\
\mathrm{~m}=\mathrm{SF}\end{array}$ & \multicolumn{4}{|c|}{$\begin{array}{c}\text { DBPSK, } \\
\mathrm{m}=1\end{array}$} & $\begin{array}{c}\text { OQPSK, } \\
m=2\end{array}$ & $\begin{array}{l}4 \mathrm{FSK}, \\
\mathrm{m}=2\end{array}$ & $\begin{array}{l}\text { GFSK, } \\
\mathrm{m}=1\end{array}$ & $\begin{array}{c}\text { GMSK } \\
\mathrm{m}=1\end{array}$ \\
\hline $\begin{array}{l}\text { Помехоустойчивое } \\
\text { кодирование на } \\
\text { линии "вверх" }\end{array}$ & $\begin{array}{l}4 / 5,2 / 3 \\
4 / 7,1 / 2\end{array}$ & Н.д. & \multicolumn{2}{|c|}{$1 / 2$} & Нет & \multicolumn{3}{|c|}{$1 / 2$} & $1 / 3$ \\
\hline $\begin{array}{l}\text { Модуляция на } \\
\text { линии "вниз" }\end{array}$ & $\begin{array}{c}\text { ЛчМ, } \\
\mathrm{SF}=6 \ldots .12 \\
\mathrm{~m}=\mathrm{SF}\end{array}$ & $\begin{array}{l}\text { GFSK, } \\
\mathrm{m}=1\end{array}$ & $\begin{array}{l}\text { BPSK, } \\
m=1\end{array}$ & \multirow{5}{*}{ Н.д. } & $\begin{array}{l}\text { BPSK, } \\
m=1\end{array}$ & $\begin{array}{c}\text { OQPSK, SF8, } \\
\mathrm{m}=2\end{array}$ & $\begin{array}{l}\text { DBPSK, } \\
\mathrm{m}=1\end{array}$ & $\begin{array}{l}\text { GFSK, } \\
\mathrm{m}=1\end{array}$ & $\begin{array}{c}\text { GMSK, } \\
\mathrm{m}=1\end{array}$ \\
\hline $\begin{array}{l}\text { Помехоустойчивое } \\
\text { кодирование на } \\
\text { линии "вниз" }\end{array}$ & $\begin{array}{l}4 / 5,2 / 3 \\
4 / 7,1 / 2\end{array}$ & Н.д. & $1 / 2,1 / 3$ & & & \multicolumn{3}{|c|}{$1 / 2$} & $1 / 3$ \\
\hline $\begin{array}{l}\text { Roll-Off на линии } \\
\text { "вниз" ("вверх") } \\
\end{array}$ & 0 & $0,14-0,28$ & Н.д. & & Нет & 0,8 & Н.д. & $0,2-0,4$ & \multirow{3}{*}{ Н.д. } \\
\hline $\begin{array}{l}\text { Предельное значе- } \\
\text { ние C/N на линии } \\
\text { "вверх", дБ }\end{array}$ & \multirow{2}{*}{ Минус 20} & $+6 \ldots+9$ & \multirow{2}{*}{+5} & & \multirow{2}{*}{+9} & Минус 17,5 & +6 & \multirow{2}{*}{+6} & \\
\hline $\begin{array}{l}\text { Предельное значе- } \\
\text { ние С/N на линии } \\
\text { "вниз", дБ }\end{array}$ & & Н.д. & & & & Минус 4,5 & +9 & & \\
\hline $\begin{array}{l}\text { Множественный } \\
\text { доступ }\end{array}$ & \multicolumn{5}{|c|}{ Aloha } & MF-TDMA & SAloha & CSMA/CA & $\begin{array}{l}\text { TS-UNB } \\
\text { Проприе- } \\
\text { тарный } \\
\end{array}$ \\
\hline $\begin{array}{l}\text { Максимальный } \\
\text { пакет "вверх", байт }\end{array}$ & \multirow{2}{*}{255} & $26-29$ & 40 & \multirow{5}{*}{ Н.д. } & 27 & \multirow{2}{*}{302} & \multirow{2}{*}{ Н.д. } & \multirow{2}{*}{256} & \multirow{2}{*}{24} \\
\hline $\begin{array}{l}\text { Максимальный } \\
\text { пакет "вниз", байт }\end{array}$ & & 28 & Н.д. & & 25 & & & & \\
\hline $\begin{array}{l}\text { Максимальный } \\
\text { объем полез- } \\
\text { ной информации } \\
\text { "вверх", байт }\end{array}$ & \multirow{2}{*}{242} & 12 & 8 & & \multirow{2}{*}{16} & \multirow{2}{*}{255} & \multirow{2}{*}{23} & 251 & $<245$ \\
\hline $\begin{array}{l}\text { Максимальный } \\
\text { объем полез- } \\
\text { ной информации } \\
\text { "вниз", байт }\end{array}$ & & 8 & $\begin{array}{c}8 \times n \\
n=1,2 \ldots 16\end{array}$ & & & & & 251 & 250 \\
\hline $\begin{array}{l}\text { Ограничение } \\
\text { интенсивности } \\
\text { пакетов } \\
\text { (или цикличность) }\end{array}$ & Менее 10\% & $\begin{array}{c}140 \\
\text { в день } \\
\text { "вверх", } \\
4 \text { в день } \\
\text { "вниз" }\end{array}$ & Н.д. & & $\begin{array}{c}\text { 0,1\% } \\
\text { "вверх" } \\
1 \% \text { "вниз" }\end{array}$ & $\begin{array}{l}\text { В соответ- } \\
\text { ствии с НТД }\end{array}$ & $\begin{array}{l}\text { В соот- } \\
\text { ветствии } \\
\text { с НТД }\end{array}$ & $\begin{array}{l}\text { В соответ- } \\
\text { ствии с НТД }\end{array}$ & $\begin{array}{l}\text { В соот- } \\
\text { ветствии } \\
\text { с НТД }\end{array}$ \\
\hline Стандарт & $\begin{array}{l}\text { LoRa Alliance } \\
\text { Открытый }\end{array}$ & $\begin{array}{l}\text { Проприе- } \\
\text { тарный }\end{array}$ & $\begin{array}{c}\text { WAWIOT } \\
\text { Проприе- } \\
\text { тарный } \\
\text { ПНСТ } \\
354-2019\end{array}$ & $\begin{array}{c}\text { СТРИж } \\
\text { Закрытый } \\
\text { проприе-- } \\
\text { тарный }\end{array}$ & $\begin{array}{l}\text { Предвари- } \\
\text { тельный } \\
\text { стандарт }\end{array}$ & $\begin{array}{l}\text { Weightless } \\
\text { Alliance } \\
\text { Открытый } \\
\text { частично }\end{array}$ & $\begin{array}{l}\text { На ста- } \\
\text { дии раз- } \\
\text { работки }\end{array}$ & $\begin{array}{l}\text { DASH7 } \\
\text { Alliance } \\
\text { условно } \\
\text { открытый }\end{array}$ & $\begin{array}{c}\text { На основе } \\
\text { TS } 103357 \\
\text { (Стандарт } \\
\text { ЕTSI) }\end{array}$ \\
\hline Модем (чип) & $\begin{array}{l}\text { SX127x } \\
\text { SX126x }\end{array}$ & $\begin{array}{l}\text { ATA8520 } \\
\text { ATA8520E } \\
\text { AX-SIGFOX } \\
\end{array}$ & AX5043 & $\begin{array}{c}\text { AX8505 } \\
2 f 143\end{array}$ & Н.д. & $\begin{array}{c}\text { Н.д. } \\
\text { Оборудо- }\end{array}$ & AX805 & $\begin{array}{c}\text { SX1282 } \\
\text { ADUCRF101 }\end{array}$ & CCl352R \\
\hline Контроллер & SX1301/1302 & STM32 & STM32 & STM32 & & $\begin{array}{l}\text { вание KOM-- } \\
\text { пании UBIIK }\end{array}$ & & $\begin{array}{l}\text { STM 32, } \\
\text { CC430 }\end{array}$ & \\
\hline
\end{tabular}


информацию предполагается короткими пакетами с низкой цикличностью.

LoRa и LoRaWAN представлены в стандарте ETSI TR 103 526. Технология LoRa обычно трактуется как физический уровень сети в совокупности с кон кретными физическими устройствами, а протокол LoRaWAN определяет правила каналообразования на всех уровнях OSI. Это наиболее распространенная открытая технология и открытый протокол, развитие и унификацию которых поддерживает LoRa Alliance $[9,10]$.

Ключевая особенность технологии LoRa - исполь зование специфических сигнально-кодовых конструкций (CKK) с расширением спектра сигнала (подобно линейной частотной модуляции - ЛЧМ), которые могут надежно передавать информацию при уровне сигналов ниже уровня шума до 20 дБ (C/N = минус 20 дБ при SF12), как на линии "вверх", так и на линии "вниз". Благодаря этому свойству обеспечивается повышенная помехозащищенность каналов передачи информации. Но расплата за это - снижение эффективности использования радиочастотного спектра. Например, скорость передачи в канале 125 кГц при самой высокой чувствительности (SF12) - примерно 250 бит/с (для такой скорости, например, при модуляции QPSK 1/2 достаточно всего 250 Гц полосы частот, то есть в 50 раз меньше, но тогда и значение C/N нужно обеспечить в 400 раз более высокое).

В то же время избыточность полосы пропускания канала относительно скорости передаваемой информации обеспечивает устойчивость технологии LoRa при возникновении эффекта Доплера в части изменения частоты сигнала (статистический эффект) и скорости его изменения (динамический эффект) [11-16]. Предел устойчивости к статическому изменению частоты, заявленный в технических описаниях чипов LoRa (SX1276/77/78/79, SX1272/73), составляет до 25\% от ширины полосы частот канала при включенном режиме оптимизации структуры пакета.

Устойчивость к эффекту Доплера в сочетании с возможностью работы ниже уровня шума - определяющее техническое свойство при выборе технологии LoRa для новых спутниковых систем IoT. Однако следует отметить, что предел устойчивости CKK LoRa к эффекту Доплера для различных сочетаний CKK окончательно пока не определен. Это предмет будущих исследований. Решение дан ${ }^{-}$ ной перспективной задачи осложняется мощным влиянием на достоверность передачи информации эффектов многолучевости на трассе распространения $[17,18]$, которые носят случайный характер.
SigFox рассматривается в [19-24]. В названии SigFox обозначается компания SIGFOX SACA (Франция), принадлежность к ее сети One global 0G и проприетарная технология SigFox. Характерная особенность технологии - использование множества сверх узких (100 Гц) каналов на линии "вверх" (от абонента к шлюзовой станции) с целью получения достаточной энергетики радиоканала. В результате пропускная способность канала менее 100 бит/с, но таких каналов сотни. Первоначально технология SigFox была однонаправленной и поддерживала связь только по линии "вверх". В последующем технология дорабатывалась для обеспечения двунаправленной связи. В канале "вниз" используется более широкая полоса частот, поскольку абонентское устройство должно быть дешевым, то есть обладать пониженной чувствительностью к изменению частоты, связанной с нестабильностью задающего генератора и перепадом температур.

Но если абонент находится в движении, технология SigFox неприменима вследствие проявления эффекта Доплера. Для обеспечения работоспособности в движении требуется организация сложных алгоритмов определения частот передачи и приема, основанных на измерении координат объекта и базовой станции (или спутника), их относительной скорости и вычислении прогноза изменения частоты. Решить эту задачу можно, но абонентское устройство заметно увеличится в цене, а его эксплуатационные показатели снизятся. Естественно, применение технологии SigFox для спутниковой системы IoT, реализуемой с использованием негеостационарных орбит, невозможно в случае прямого доступа к абонентским устройствам в диапазонах ISM. Однако есть сведения, что компания Eutelsat совместно с компанией SigFox создала и в конце 2018 года запустила демонстрационные низкоорбитальные спутники (проект ELO). Подробностей технических решений или результатов тестирования в открытом доступе нет.

NB-Fi, OpenUNB, XNB представлены в [25-28]. Технологии XNB и NB-Fi - производные от технологии SigFox. XNB поддерживается в сети компании "Стриж", а NB-Fi - в сети компании WAVIOT. Этим проприетарным технологиям присущи все свойства SigFox. Следует отметить, что в 2019 году протокол беспроводной передачи данных на основе узкополосной модуляции радиосигнала NB-Fi оформлен в России как предварительный национальный стандарт (ПНСТ 354-2019) сроком действия до апреля 2022 года. Но описание технологий и протоколов на физическом и канальном уровнях отрывочно и не раскрывает сути технологии и протоколов. 
В 2019 году появился еще один проект российского предварительного стандарта, посвященный технологии под названием OpenUNB [26, 27]. Несмотря на содержащиеся в материале неточности и ошибки (https://www.pvsm.ru/cat/openunb), можно сделать вывод, что это очередная интерпретация технологии SigFox с попыткой компенсации нестабильности кварцевого генератора абонентского устройства.

В отличие от NB-Fi и OpenUNB, разработчики технологии XNB придерживаются принципа полностью закрытого проприетарного протокола. В качестве элементной базы в XNB и NB-Fi применяются чипы, по предварительным оценкам, тех же производителей, что и в оборудовании сети SigFox. Для OpenUNB данные не опубликованы.

Таким образом, XNB, NB-Fi и OpenUNB - технологии, подобные SigFox, не могут служить базовыми для использования в спутниковой системе IoT.

Dash7, Mioty, SNBWAN представлены в [29-37]. В отличие от технологий типа SigFox и LoRa, они основаны на синхронной передаче данных (приемнику и передатчику известно время передачи данных). Спектральная эффективность таких технологий заметно выше, чем технологии LoRa. To есть при одной и той же полосе частот канала потенциальное число подключений может быть больше в разы. Но в результате возникает проблема энергетического потенциала радиолинии, поскольку требуется достигать $\mathrm{C} / \mathrm{N}$ не менее плюс 5-6 дБ для устойчивой работы (в отличие от технологии LoRa сигнал должен значительно превышать шумы в канале связи).

Кроме того, возникает проблема компенсации эффекта Доплера. Она решаема (как и при использовании SigFox), но этот алгоритм для спутниковой сети IоТ приводит к тому, что стоимость абонентского устройства значительно увеличивается за счет необходимости применения приемника GPS/ГЛОНАСС, который требуется для вычисления частотных уходов и скорости изменения частоты при определении требуемой рабочей частоты канала и его частотной подстройки. Наряду с этим необходимо постоянно обновлять альманах всех спутников низкоорбитальной орбитальной группировки и передавать эти данные на абонентские устройства. Соответственно, на спутник должны постоянно загружаться обновленные служебные данные и со спутника транслироваться абонентам, а абонент должен хранить альманах, обновлять его и выполнять вычисления. Объем служебной информации резко возрастает, что сводит на нет преимущества спектральной эффективности этих технологий, а при быстром или динамичном движении абонента такой процесс может оказаться невозможным. Указанные проблемы исключают применение технологий на основе синхронной передачи данных для спутниковой сети IoT, требующей максимально невысокой стоимости и предельно низ кого уровня потребления абонентских устройств.

Следует отметить интересную специфику технологий Mioty и DASH7. Так, особенность протокола Mioty заключается в том, что для передачи объемного пакета, например, нескольких сотен байт, его предварительно сегментируют на мелкие пакеты (предположительно по 12 байт), и каждый мелкий пакет передается на своей частоте. В результате снижается вероятность коллизии пакетов (по оцен ${ }^{-}$ кам разработчиков технологии, выигрыш составляет примерно 20\% по отношению к слотированной Alohe).

Разработка технологии DASH7 была инициирована Министерством обороны США и альянсом НАTO. В 2009 году Министерство обороны США выделило частной промышленности 429 млн долл. для развития DASH7, что имело большое значение для коммерциализации и стандартизации технологии. В итоге DASH7 была разработана компанией Savi Technology (2010 год) и впервые применена в ходе войны в Персидском заливе. В последующем технология DASH7 развивалась в направлении ее коммерциализации и расширения функций путем взаимодействия с метками RFID как с оконечными устройствами.

Однако эффект Доплера не позволяет адаптировать эти технологии к низкоорбитальной спутниковой системе ІоТ с сохранением конкурентоспособных ценовых параметров абонентских устройств.

Weightless-P рассматривается в [38]. Технология Weightless подразумевает три стандарта: Weightless-N, Weightless-W и Weightless-P. Обратимся к варианту Weightless-P (первая версия стандарта Weightless-Р появилась только в 2017 году), который обеспечивает наиболее высокую энергетику каналов. Технология отличается от обычных технологий синхронной передачи возможными вариан тами CKK, которые предусматривают расширение спектра в четыре или восемь раз по отношению к минимально требуемой полосе частот для максимальной битовой скорости при модуляции OQPSK с $\mathrm{FEC}=1 / 2$. Соответственно спектральная эффективность пропорционально снижается, как и в технологии LoRa, но благодаря этому появляется возможность принимать и передавать информацию ниже уровня шумов.

Стоит отметить, что на линии "вниз" полоса частот расширена до 100 кГц. Это дает возможность 
работать в движении при наличии достаточно простых оконечных устройств и минимальном значении $\mathrm{C} / \mathrm{N}=-4,5$ дБ, что все же примерно на 12,5 дБ больше, чем на линии "вверх".

Тем не менее технология Weightless-P не получила широкого распространения.

Применение этой технологии для спутниковой сети ІоТ технически возможно, но экономически неэффективно, поскольку элементная база не представлена широко на открытом рынке.

\section{ВЗАИМОСВЯЗЬ ПАРАМЕТРОВ}

К ключевым характеристикам СКК обычно относятся спектральная и энергетическая эффективность, а также помехоустойчивость. Эти показатели связаны с пороговым отношением сигнал/ шум $(\mathrm{C} / \mathrm{N})$ в канале с аддитивным белым гауссовским шумом (АБГШ). Шеннон показал, что потенциальная скорость R в канале с АБГШ определяется достигнутым значением $\mathrm{C} / \mathrm{N}$ и шириной его шумовой полосы пропускания W. Причем $\mathrm{R}_{\mathrm{b}}$ всегда менее битовой скорости в канале R:

$$
\mathrm{R}_{\mathrm{b}}<\mathrm{R}=\mathrm{W} \log _{2}(1+\mathrm{C} / \mathrm{N}) .
$$

Степень приближения $\mathrm{R}_{\mathrm{b}} \mathrm{K} \mathrm{R}$ - обобщенная характеристика эффективности СКК.

Скорость в физическом канале, в котором информация передается цифровыми многопозиционными методами манипуляции (фазы, частоты, амплитуды), характеризуется числом битов, передаваемых в секунду (с учетом коэффициента расширения спектра, если применяется).

Вероятность ошибочного приема бита информации $\left(\mathrm{P}_{\mathrm{b}}\right)$ определяется достигнутым значением отношения энергии бита $\left(\mathrm{E}_{\mathrm{b}}\right)$ к спектральной плотности мощности шума $\left(\mathrm{N}_{\mathrm{o}}\right)$ в канале. Если принять, что $\mathrm{P}_{\mathrm{b}}$ стремится к нулю, можно записать взаимосвязь:

$$
\mathrm{C} / \mathrm{N}=\mathrm{E}_{\mathrm{b}} / \mathrm{N}_{\mathrm{o}}(\mathrm{R} / \mathrm{W}) \text {. }
$$

Тогда соотношение Шеннона примет вид:

$$
\mathrm{R}_{\mathrm{b}}<\mathrm{W} \log _{2}\left(1+\mathrm{E}_{\mathrm{b}} / \mathrm{N}_{\mathrm{o}}(\mathrm{R} / \mathrm{W})\right) .
$$

В пределе при $\mathrm{R} / \mathrm{W} \rightarrow 0$ значение $\mathrm{E}_{\mathrm{b}} / \mathrm{N}_{\mathrm{o}}=0,693$ или минус 1,6 дБ независимо от значения C/N. Это значение называют пределом Шеннона.

Значение $\mathrm{R}_{\mathrm{b}}$ взаимосвязано с параметрами используемого типа CKK:

$$
\mathrm{R}_{\mathrm{b}}=\mathrm{R}_{\mathrm{s}} \mathrm{mFEC},
$$

где $\mathrm{R}_{\mathrm{s}}$ - символьная скорость;

$\mathrm{m}$ - число бит в одном символе;

FEC - коэффициент помехоустойчивого кодирования.

Из (2) и (4) следует оценка взаимосвязи значения $\mathrm{C} / \mathrm{N}$, используемой CKК и значения $\mathrm{E}_{\mathrm{b}} / \mathrm{N}_{0}$ :

$$
\mathrm{C} / \mathrm{N}=\mathrm{E}_{\mathrm{b}} / \mathrm{N}_{\mathrm{o}} \mathrm{m} \text { FEC }\left(\mathrm{R}_{\mathrm{s}} / \mathrm{W}\right) .
$$

Для СКК без расширения спектра $\mathrm{R}_{\mathrm{s}}=\mathrm{W} /(1+\mathrm{r})$. В случае расширения спектра, например для CKK LoRa, получаем $\mathrm{m}=\mathrm{SF}, \mathrm{R}_{\mathrm{s}}=\mathrm{W} / 2^{\mathrm{SF}}$, где FEC $=4 /(4+\mathrm{CR})$, $\mathrm{CR}=1,2,3$, 4. Таким образом, скорость передачи информации в канале с использованием CKK LoRa снижается при увеличении SF:

$$
\mathrm{R}_{\mathrm{b}}=\mathrm{SF} W / 2^{\mathrm{SF}} \text { FEC. }
$$

За счет расширения спектра прием информации при использовании CKK LoRa обеспечивается при уровне сигнала значительно ниже уровня шумов в канале. Для CKK LoRa соотношение $\mathrm{R} / \mathrm{W}>\mathrm{R}_{\mathrm{b}} / \mathrm{W}<<1$. Из (5) следуют значения C/N для CKK LoRa при различных величинах SF и заданном коэффициенте $\mathrm{FEC} \mathrm{при} \mathrm{условии} \mathrm{R}_{\mathrm{b}}=\mathrm{R}$ :

$$
\mathrm{C} / \mathrm{N}=\mathrm{E}_{\mathrm{b}} / \mathrm{N}_{\mathrm{o}}\left(\mathrm{R}_{\mathrm{b}} / \mathrm{W}\right)=\mathrm{E}_{\mathrm{b}} / \mathrm{N}_{\mathrm{o}}\left(\mathrm{SF} / 2^{\mathrm{SF}}\right) \mathrm{FEC},
$$

где $\mathrm{R}_{\mathrm{b}}=\mathrm{R}_{\mathrm{s}} \mathrm{m}$ FEC $=\mathrm{SF} \mathrm{W} / 2^{\mathrm{SF}} \mathrm{FEC}-$ битовая скорость в канале с CKK LoRa на физическом уровне.

В литературе часто приводятся значения чувствительности приемника. На этом основании сопоставляются технологии LPWAN. Но конечный результат сравнения зависит от достигнутого значения коэффициента шума приемника и полосы частот канала:

$$
\mathrm{C} / \mathrm{N}=\mathrm{S}+175-10 \lg \mathrm{W}-\mathrm{NF},
$$

где $\mathrm{S}$ - чувствительность приемника, дБм; NF- коэффициент шума приемника, дБ.

Следует отметить, что указанные выше соотношения взаимосвязи $\mathrm{C} / \mathrm{N}$ и $\mathrm{E}_{\mathrm{b}} / \mathrm{N}_{\mathrm{o}}$ предполагают равенство $\mathrm{P}_{\mathrm{b}}$.

\section{Зависимость $\mathrm{C} / \mathrm{N}$ и $\mathrm{E}_{\mathrm{b}} / \mathrm{N}_{\mathrm{o}}$ от вероятности ошибки на бит}

Зависимости $\mathrm{E}_{\mathrm{b}} / \mathrm{N}_{\mathrm{o}}\left(\mathrm{P}_{\mathrm{b}}\right)$ для типичных СКК с фазовой или частотной манипуляцией хорошо известны и представлены во многих источниках. Для CKK LoRa функция $\mathrm{E}_{\mathrm{b}} / \mathrm{N}_{\mathrm{o}}\left(\mathrm{P}_{\mathrm{b}}\right)$ в явном виде неизвестна.

Из (7) следуют предельные значения C/N для CKK LoRa при заданном FEC (табл.2), вытекающие 
Таблица 2. Типовые параметры CKK LoRa для канала 125 кГц

\begin{tabular}{|c|c|c|c|c|c|c|c|}
\hline \multirow[t]{2}{*}{ LoRa } & \multirow[t]{2}{*}{$\mathrm{SF}=\mathrm{m}$} & \multirow[t]{2}{*}{$M=2^{m}$} & \multirow{2}{*}{$\begin{array}{c}\mathrm{R}_{\mathrm{b}}, 6 и т / \mathrm{c} \\
\mathrm{c} \mathrm{FEC}=4 / 5\end{array}$} & \multirow{2}{*}{$\begin{array}{c}\text { C/N, дБ } \\
\text { при } \mathrm{FEC}=4 / 5 \\
\mathrm{P}_{\mathrm{b}}=10^{-4}\end{array}$} & \multirow{2}{*}{ 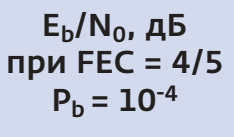 } & $E_{b} / N_{0}$, дБ & $\mathrm{C} / \mathrm{N}$, дБ \\
\hline & & & & & & \multicolumn{2}{|c|}{ Предел Шеннона } \\
\hline DRO & 12 & 4096 & 293 & $-21,2$ & 5,1 & $-1,593$ & $-27,9$ \\
\hline DRI & 11 & 2048 & 537 & $-18,4$ & 5,3 & $-1,593$ & $-25,3$ \\
\hline DR2 & 10 & 1024 & 977 & $-15,6$ & 5,5 & $-1,593$ & $-22,7$ \\
\hline DR3 & 9 & 512 & 1758 & $-12,7$ & 5,8 & $-1,593$ & $-20,1$ \\
\hline DR4 & 8 & 256 & 3125 & $-9,9$ & 6,1 & $-1,593$ & $-17,6$ \\
\hline DR5 & 7 & 128 & 5469 & $-7,1$ & 6,5 & $-1,593$ & $-15,2$ \\
\hline
\end{tabular}

из предела Шеннона. Их можно трактовать как зна чения $\mathrm{C} / \mathrm{N}$, при которых вероятность приема сигнала 0,5, то есть достоверный и ошибочный прием сигнала равновероятны.

В [39] представлены результаты моделирования канала LoRa при передаче пакетов PHY payload 13 байт с использованием имитационных программ NS-3. На основе этих результатов и предложено эмпирическое соотношение, определяющее зависимость вероятности ошибки приема бита информации (в диапазоне $\mathrm{P}_{\mathrm{b}}$ до 10-6) в зависимости от значения C/N для различных SF и FEC в канале с полосой 125 кГц:

$$
\operatorname{Lg} P_{b}=\alpha \exp (\beta C / N)
$$

где $\alpha$ и $\beta$ - коэффициенты аппроксимации при моделировании.

Соответствующие зависимости $\mathrm{C} / \mathrm{N}\left(\mathrm{P}_{\mathrm{b}}\right)$ приведены на рис.1. Результаты примерно совпадают с [17] для частных примеров. Но следует учитывать, что полученные зависимости $\mathrm{C} / \mathrm{N}\left(\mathrm{P}_{\mathrm{b}}\right)$ справедливы в отсутствие многолучевого распространения. Наличие отражений и переотражений на трассе распространения сигнала могут существенно повлиять (ухудшить $\mathrm{C} / \mathrm{N}$ в канале до 20 дБ) на конечный результат [17, 18], что в общем случае является случайным процессом.

B отличие от CKK LoRa в иных технологиях LPWAN используются CKK на основе модификаций двух- или четырехпозиционной фазовой или частотной манипуляции. Значение $\mathrm{R}_{\mathrm{b}}$ в этом случае определяется как (4), но важно учитывать крутизну амплитудно-частотного параметра фильтра при обработке сигнала, которая характеризуется коэффициентом roll-off (r), поэтому $\mathrm{R}_{\mathrm{s}}=\mathrm{W} /(1+\mathrm{r})$.

\section{ЗАКЛЮЧЕНИЕ}

Бесспорное достоинство спутниковых систем связи - их глобальность (или почти глобальность). Но любая спутниковая система связи не будет востребована на массовом рынке, если применяемые абонентские устройства не обеспечивают удобство эксплуатации, а их ценовые параметры в сочетании с ценовыми показателями предоставляемых услуг не отвечают условиям конкурентоспособности с наземными сетями.

Конкурентоспособность спутниковых систем можно значительно повысить, если адаптировать технологии, массово применяемые в наземных сетях, к особенностям спутниковых сетей. В данном случае эта задача рассмотрена применительно к спутниковым сетям IоT.

Результаты анализа технологий LPWAN показывают, что технология LoRa - наилучший вариант для применения в низкоорбитальной спутниковой системе с целевой функцией IоT. В этом случае полезная нагрузка спутника представляет собой ретрансляционную аппаратуру с обработ кой и регенерацией сигналов. В результате обеспечивается высокая гибкость выбора параметров для достаточного энергетического потенциала радиолиний при приеме-передаче коротких пакетов с использованием типовых модемов LoRa, применяемых на массовом рынке IоT в абонентских устройствах LPWAN LoRa.

Представленные в статье зависимости $\mathrm{C} / \mathrm{N}\left(\mathrm{P}_{\mathrm{b}}\right)$ позволяют выполнить уточненный расчет 

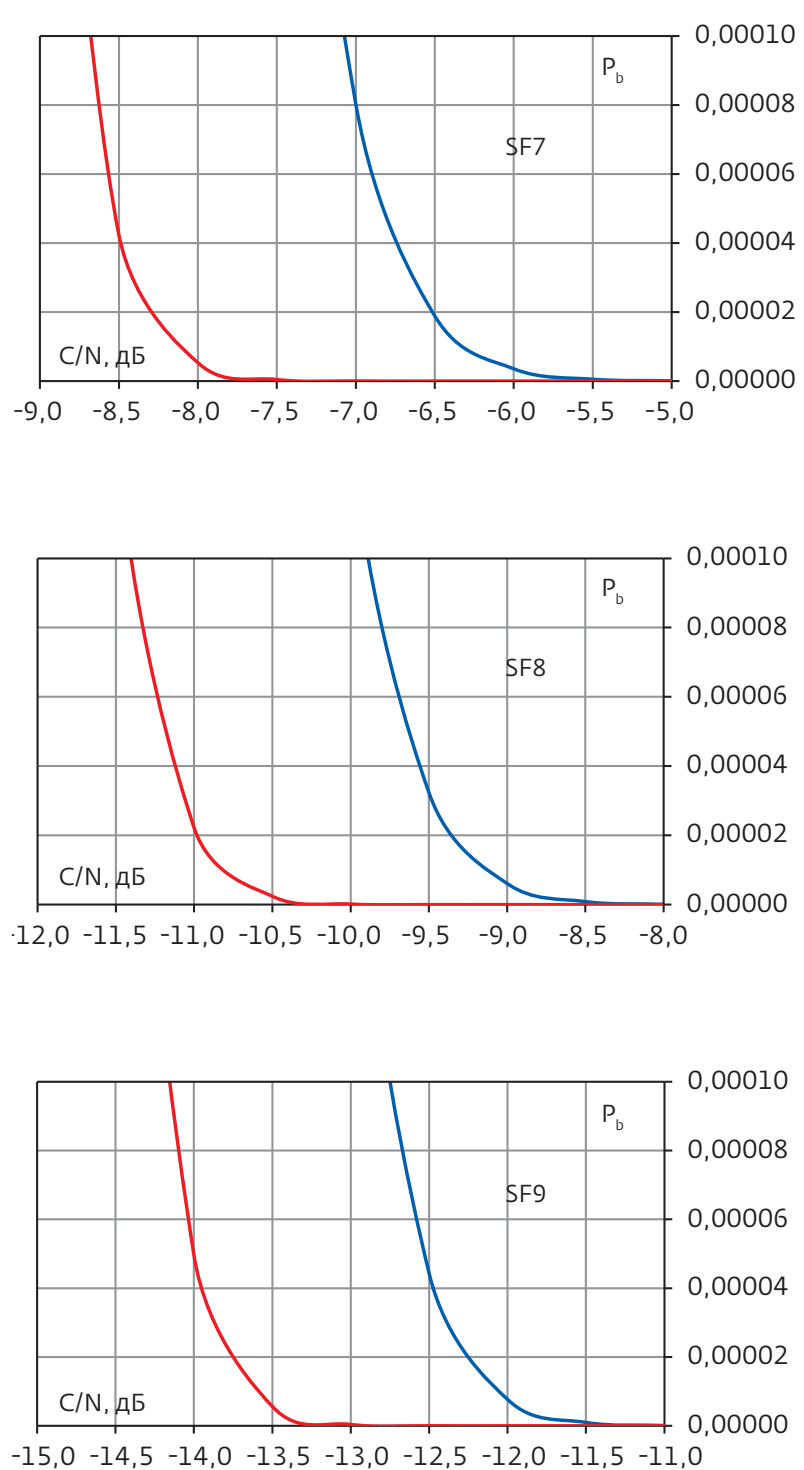
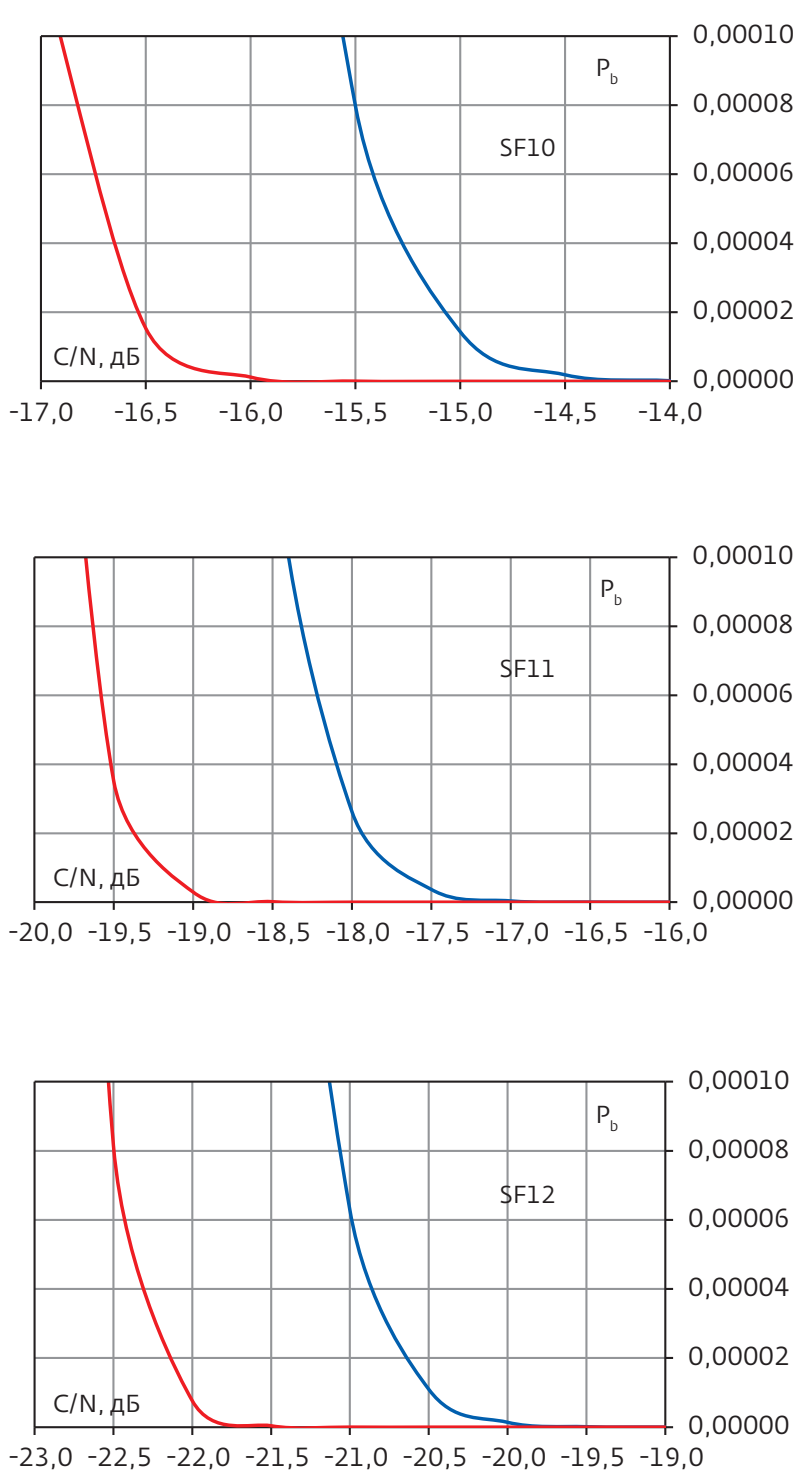

Рис.1. Зависимости значения C/N в канале с АБГШ 125 кГц от вероятности ошибки на бит при FEC = 4/5 (синие кривые) и 4/7 (красные кривые) для CKK LoRa

бюджета абонентских радиолиний. При использовании диапазона ISM 868/915 МГц на линии "космос - Земля" необходимо учитывать ограничения на эквивалентную изотропно излучаемую мощность спутниковых каналов для исключения помех наземными системами LPWAN и SDR [40].

Следует отметить, что CKK LoRa обладает повышенной устойчивостью к эффекту Доплера, но пределы этой устойчивости требуют уточнения.

\section{ЛИТЕРАТУРА}

1. Анпилогов В.Р. Глобальные спутниковые системы M2M/IоT: новые проекты и новый рынок. V Международная конференция "Инжиниринг \& Телекоммуникации - En\&T 2018", 15 ноября 2018 года, http://2019.ent.info/old/roundtableru.shtml

2. Анпилогов В. Обзор докладов и обсуждений на круглом столе $\mathrm{V}$ Международной конференции "Инжиниринг \& Телекомму- 
никации - EN\&T 2018" // Технологии и средства связи. 2018. № S1. Специальный выпуск "Спутниковая связь и вещание 2019". С. 32-38.

3. Эйдус А.Г. Анализ действующих негеостационарных спутниковых систем на рынке M2M/Іот и оценка коммерческой перспективности планируемых многоспутниковых систем // Технологии и средства связи. 2017. № 6. Специальный выпуск "Спутниковая связь и вещание 2018". С. 20-27.

4. M2M and IoT via Satellite, 10th Edition. September 2019, NSR.

5. Liu J., Li Y., Wang X., Chen Sh. A hybrid global-local load balancing routing scheme for the Internet of Things through satellite networks // International Journal of Distributed Sensor Networks. 2017. V. 13(3). P. 1-16.

6. Агаджанов М. Российские операторы связи приступили к тестированию сим-карт NBIoT для Интернета вещей. 25.10.2018, https:// habr.com/ru/post/427643/

7. Maria Rita Palattella, Nicola Accettura. Enabling Internet of Everything Everywhere: LPWAN with satellite backhaul. 2018 Global Information Infrastructure and Networking Symposium (GIIS), October 2018, Thessaloniki, Greece. P. 1-5.

8. Mekki K., Bajic E., Chaxel F., Meyer F. A comparative study of LPWAN technologies for large-scale IoT deployment, ICT Express 5. 2019. P. 1-7.

9. Обзор технологии LoRa, https://itechinfo.ru/ node/46

10. LoRa Alliance, https://lora-alliance.org/

11. Petjjrvi J., Mikhaylov K., Pettissalo M., Janhunen J., and Iinatti J. Performance of a lowpower wide-area network based on LoRa technology: Doppler robustness, scalability, and coverage, International Journal of Distributed Sensor Networks. 2017. V. 13. № 3.

12. Colavolpe G., Foggi T., Ricciulli M.A., Zanettini Y.J., Alameda M. Reception of LoRa Signals From LEO Satellites, IEEE Transactions on aerospace and electronic systems. V. 55. December 2019. P. 3587-3602.

13. Doroshkin A., Zadorozhny A., Kus O., Prokopyev V., Prokopyev Y. Laboratory testing of LoRa modulation for CubeSat radio communications VI International forum for young scientists Space Engineering 2018. P. 1-5.

14. Jung Suk Joo. A study on the interleaving scheme of LoRa PHY layer // International Re- search Journal of Computer Science (IRJ CS). Issue 01 . V. 7. January 2020. P. 6-10.

15. Adelantado Ferran, Vilajosana Xavier, Tuset-Peiro Pere, Martinez Borja, Melia-Segui Joan, and Watteyne Thomas. 2017. Understanding the limits of LoRaWAN. IEEE Commun. Mag. 55, 9 (2017). P. 34-40.

16. Doroshkin A.A., Zadorozhny A.M., Kus O.N., Prokopyev V.Y., Prokopyev Y.M. Experimental Study of LoRa Modulation Immunity to Doppler Effect in CubeSat Radio Communications, IEEE Access, June 24, 2019.

17. Elshabrawy T., Robert J. Analysis of BER and Coverage Performance of LoRa Modulation under Same Spreading Factor Interference Conference: 2018 IEEE 29th Annual International Symposium on Personal, Indoor and Mobile Radio Communications (PIMRC). Sep tember 2018.

18. Dias C.F., de Lima E.R., Fraidenraich G. Bit Error Rate Closed-Form Expressions for LoRa Systems under Nakagami and Rice Fading Channels. Sensors 2019. 19, 4412 https://www. mdpi.com/journal/sensors

19. Zuniga J.C., Ponsard B. SIGFOX System Description, LPWAN Working Group, SIGFOX Ex pires: September 14. 2017.

20. https://www.sigfox.com/en

21. Gomez C., Veras J.C., Vidal R.l., Casals L., Paradells J. A Sigfox Energy Consumption Model, Sensors 2019. 19, 681 https://www. mdpi.com/journal/sensors

22. Rodriguez I., Lauridsen M., Arvidsson K., Kvarnstrand J., Andersson M., Mogensen P. Testing of Low-Power Wide-Area Technologies in Controlled Propagation Environments, Loughborough Antenna and Propagation Conference 2017.

23. Abu Hemjal: SigFox based Internet of Things: Technology, Measurements and Development, Master of Science thesis Tampere University Master's Degree Programme in Electrical Engineering, May 2019.

24. Lavric A., Petrariu A.I., Popa V., Long Range SigFox Communication Protocol Scalability Analysis Under Large-Scale, HighDensity Conditions, IEEE Access. V. 7. 2019. P. 35816-35825.

25. Протокол беспроводной передачи данных на основе узкополосной модуляции радиосигнала (NB-Fi), Предварительный национальный стандарт Российской Федерации. ПНСТ 354-2019. 
26. Протокол передачи данных для высокоемких сетей на основе сверхузкополосной модуляции радиосигнала, https://iot.skoltech. ru/2019/07/29/tsk-nti-pri-skoltehe-vylozhil-votkrytyj-dostup-novyj-standart-openunb-dlyainterneta-veshhej/OpenUNB

27. Обсуждение стандарта openUNB, https:// habr.com/ru/post/464103/

28. CPT: Технология узкополосной связи LPWAN XNB, https://www.tadviser.ru/index.php/

29. DASH7 Alliance Wireless Sensor and Actuator Network Protocol, https://dash7-alliance.org/

30. Towards IP over LPWANs technologies: LoRaWAN, DASH7, NB-IoT, EEE, 2018. Sixth International Conference on Digital Information, Networking, and Wireless Communications (DINWC 2018). P. 43-47.

31. Considerations for low-power communication in industrial IoT Applications. DASH7 Alliance. October 2019. V. 1.0.

32. Mioty Alliance, https://mioty-alliance.com/

33. Mioty Alliance, https://behrtech.com/mioty/

34. Fraunhofer Institute for Integrated Circuits IIS, https://www.iis.fraunhofer.de/en/ff/lv/net/telemetrie.html
35. Short Range Devices; Low Throughput Networks (LTN); Protocols for radio interface A, ETSI TS 103357 V1.1.1 (2018-06).

36. Зверев Б., Сартаков А. SNB - новая LPWANтехнология Интернета вещей с высокой пропускной способностью, Control Engineering Россия IIoT, июнь 2019, https://controlengrussia.com/internet-veshhej/snb/

37. Зверев Б., Сартаков А. SNB - новая LPWANтехнология Интернета вещей с непревзойденной пропускной способностью // Беспроводные технологии. 2018. № 4, https:// wireless-e.ru/

38. Weightless-P System Specification, V. 1.037 November 2017, http://www.weightless.org/ about/weightless-specification

39. Van den Abeele F., Haxhibeqiri J., Moerman I., Hoebeke J. Scalability analysis of large-scale LoRaWAN networks in ns-3 // Journal of latex class files, V. 14, № 8, August 2015. P. 1-12.

40. Анпилогов В.Р., Нгуен Д.А. Анализ совместимости спутниковых сетей IoT с устройствами SRD и LPWAN в диапазонах частот 868/915 МГц // Электросвязь. 2020. № 1. C. 37-43.

\section{1-22 октября 2020}

отель «Холидей Инн Лесная», Москва, Лесная ул., д. 15

XI Международная конференция

\section{Transport Networks Russia \& CIS}

\author{
Развитие \\ телекоммуникационных транспортных сетей \\ в эпоху цифровой экономики, \\ распределенных дата-центров \\ и облачных услуг накануне запуска $5 G$
}

- Более 400 участников

- Более 50 докладчиков - операторов, регуляторов и поставщиков оборудования и сервисов

- Более 20 экспонентов

- Более 40 инфопартнеров

- Поддержка ведущих отраслевых ассоциаций и объединений деятелей рынка

Организатор:

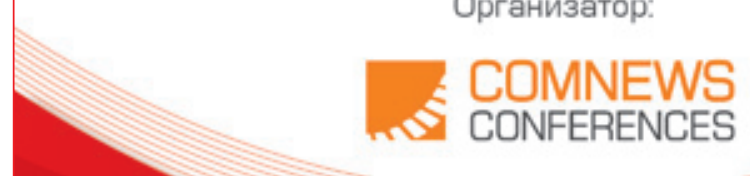

